\title{
GUIDELINES FOR ASSESSING PARENTAL PREFERENCE IN CHILD-CUSTODY DISPUTES
}

\author{
RICHARD A. GARDNER, M.D. \\ Journal of Divorce \& Remarriage, 1999, 30(1/2):1-9
}

Since the early 1960s I have been developing guidelines for legal and mental health professionals to utilize when conducting an assessment in the context of child-custody disputes (Gardner, 1982, 1986, 1989, 1992, and 1998). Over the years, I have seen attempts to quantify such evaluations with scales and score sheets. This approach is particularly attractive to those who hold that such instruments add a certain degree of "objectivity" to such assessments, objectivity that is not apparent when one uses evaluative formats in which there are no scores or formal quantification. I believe that guidelines that provide the evaluator with specific relevant areas to focus on can be useful. In the state of Michigan, a set of such guidelines is not only widely used but courts are required to follow them when making decisions in child-custody cases. The Michigan guidelines (1993) require the evaluator to compare the parents on 12 factors that are considered to be applicable to a custody/visitation determination regarding what is in the best interests of the child. Although I consider there to be some weaknesses and omissions in the Michigan criteria, I believe the principle is a sound one. Especially attractive to me is the requirement that the parents be compared on each of the items, following which the examiner comments on which parent has been preferred on each of the items and then tallies the total score for each parent.

In this report, I utilize the same principle but provide what I consider to be a superior set of guidelines, guidelines that expand upon what I consider to be the valid criteria in the Michigan protocol and omit (in part or in toto) what I consider to be the weaker criteria. Elsewhere (1999), I have published on these guidelines.

\section{1) The Stronger, Healthier Psychological Bond.}

This is first criterion because I consider it the most important. The best-interests-of-the-child presumption is too vague to be particularly useful. Everyone waves that flag: both parents, both lawyers, the judge, the guardian-ad-litem, and the parade of mental health professionals who testify on both sides. No one claims not to subscribe to this principle. No one claims that he (she) is operating against the best interests of the child. What usually happens is that all these vociferous proponents of this principle cancel each other out. Accordingly, the best-interests-of-the-child guideline is meaningless. Actually, what we are really trying to assess in a child-custody dispute is which parent has the stronger and healthier psychological bond with the child. Many aspects of the evaluation provide data of use in making this specific assessment. Joint interviews can be particular useful here, e.g., father plus child, mother plus child, both parents plus child. Particularly useful are interviews in which both parents are seen together in which they have the opportunity to confront one another directly with their often conflicting opinions regarding a whole series of events. 
When implementing this particular criterion, I utilize the following principles:

a) Preference should be given to that parent (regardless of gender) with whom the child has developed the stronger, healthier psychological bond.

b) That parent (regardless of gender) who was the primary caretaker during the earliest years of the child's life is more likely to have developed the stronger, healthier psychological bond.

c) The longer the time gap between the earliest years of the child's life and the time of the custody evaluation or decision, the greater the likelihood other factors will operate that may tip the balance in either direction regarding parental capacity.

It is important to note that this criterion is only applicable when the bonding being assessed is healthy. Obviously, pathological bonds can exist between a parent and child, but their presence would not argue for primary parental designation. Rather, their presence would often argue against such designation.

\section{2) Parenting Capacity}

This criterion focuses on the comparative ability of the parents to properly and effectively raise the children. It includes knowledge of child-rearing techniques and the utilization of humane and reasonable disciplinary measures. It includes the knowledge of how to provide the children with guidance, instruction, and care.

\section{3) Values and Morality}

Parents serve as models for their children. Accordingly, a parent with unhealthy values and/or immoral behavior is likely to transmit these undesirable qualities to the children. The evaluator assesses here honesty, sensitivity to the feelings of others, social commitment, lifestyle, and other personality qualities which would be useful for the child to emulate and identify with.

\section{4) Availability}

The evaluator should give consideration to the availability of each of the parents who are involved in the child's life. Particularly important is each individual's availability for getting the child off to school, being available on their return, and being available to care for the child during illnesses and emergency situations. This criterion also refers to availability to care for the child during school holidays and during the evening for homework assistance. 


\section{5) Educational Commitment (Curricular and Extracurricular)}

The evaluator should compare the parents with regard to their genuine commitment to the educational process and the appreciation of its importance in the child's life. Such commitment not only manifests itself by what the parent says but, more importantly, by what the parent does with regard to his (her) actual involvement in the child's school activities. This includes parent-teacher conferences, PTA meetings, as well as attendance at school extracurricular activities, e.g. sports, music and dance recitals, plays, etc. The evaluator does well to try to determine the degree of pride and joy each parent has when observing the child's involvement in the extracurricular activities.

\section{6) Health Care (Physical and Mental)}

The evaluator should compare each parent's commitment to and availability for involvement in the child's health care. This includes visits to health care providers, both physical and mental, e.g., pediatricians, psychologists, and psychiatrists. It also involves availability and commitment to the child when physically ill and home care is required.

\section{7) Appreciation of the Role of the Other Parent in the Child's Upbringing}

The evaluator should compare each parent's appreciation of the importance of the other parent in the child's upbringing. Parents who try to exclude the other parent from the child's life exhibit a parental impairment. This is especially the case if such exclusions are associated with attempts to denigrate the other parent to the point that the child may develop complete alienation from the targeted parent (Gardner, 1992, 1998).

\section{8) Cooperation}

The evaluator should compare the parents regarding the willingness to cooperate with the other parent concerning issues relevant to the healthy growth and development of the child. Although divorce hostilities may interfere with such cooperation, healthy and committed parents recognize that it is important to separate their own interpersonal difficulties from the important obligation that both of them have to cooperate in the raising of the child. Although angry and irritated with one another regarding unresolved divorce issues, healthy and committed parents are still able to cooperate with one another regarding the care of the child. Parents who require court orders before cooperating with the other parent on issues related to the child's healthy growth and development are exhibiting a parenting impairment.

\section{9) Communication}

The evaluator should compare the parents with regard to their receptivity to communicating openly and freely with one another on issues relevant to the growth and development of the child. Parents who refuse to speak on the phone with one another exhibit a parenting deficiency and this is especially the case for parents who insist on communicating only through lawyers. 


\section{0) Commitment to Providing the Child with Food, Clothing, and Shelter}

The evaluator should compare the parents with regard to their commitment to providing the child with the basic necessities of life: food, clothing, and shelter. This criterion has less to do with the actual amount of money the parent earns and more to do with the sense of commitment to providing the children with these necessities of life.

\section{1) Physical Health of Each Parent}

Obviously, in order to care properly for the child, the parent must be reasonably healthy physically. Accordingly, the examiner should get information in this realm in the course of conducting the evaluation.

\section{2) Psychological Health of Each Parent}

Each parent should be assessed psychologically to ascertain the presence of significant psychological disorders. It is not necessary that formal psychological tests be administered to each parent in the context of a child-custody evaluation. Generally, clinical information obtained from each parent about himself (herself) and about the other party will provide the necessary data. One is particularly interested in obtaining information about severe forms of psychopathology, especially when hospitalization has been required. Accordingly, the examiner should assess for evidences of psychosis, severe personality disorder, substance abuse, abuse of family members (emotional, physical, or sexual), psychopathy, and difficulties with law-enforcement officials. Relatively mild forms of psychopathology should not generally be given significant consideration here because both parents are likely to have some diagnosis and it is not generally useful to compare whether mild psychopathology category A makes one less capable as a parent than having a mild form of psychopathology B.

\section{3) Presence of Parental Surrogates in Each Parent's Home}

The evaluator should determine whether parental surrogates are living in or immediately available to the homes in which the child lives. These would include stepparents, new partners or companions, grandparents, and other individuals who are significantly involved in the child's life and would be reasonably available on a continuing basis to be involved in the child's care. Although housekeepers and maids may be given consideration in the assessment of this criterion, the evaluator should appreciate that many such individuals may be transient in the child's life.

\section{4) Appreciation of the Dangers of the Child's Exposure to and Embroilment in the Parental Conflict}

Healthy parents recognize that it is important not to expose the child to or embroil the child in the parental conflict. In contrast, parents who utilize the child in their conflict, e.g., as a weapon, as a rope in a tug of war, as a spy, and as a saboteur, are exhibiting a parental deficiency. Parents who induce in the child a campaign of denigration against the other parent, utilizing the child then as a weapon in the parental conflict also represent a significant parenting deficiency. 


\section{5) Commitment to the Child's Enrichment}

This indicator focuses on commitment to the child's involvement in activities beyond those provided in the school (both curricular and extracurricular). This would include a wide variety of enrichment activities including music lessons, sports, scouting, camping, gymnastics, travel, visits to museums and historical sites, and other forms of intellectual and emotional stimulation.

\section{6) Extended Family Involvement}

Healthy parents recognize that it is important for the child to have a sense of family ties, an appreciation of one's importance in the family network. This not only includes the extended family of the parent himself (herself) but also the extended family of the estranged spouse. Parents who expand their acrimony from the spouse to the spouse's extended family manifest a parenting deficiency.

\section{7) Involvement with the Child's Friends}

Healthy parents recognize the important of friends in a child's life. Accordingly, they facilitate visits by friends to the home and the child's visiting friends in other homes. They also facilitate overnight visits as well. Accordingly, the evalutor should compare the parents with regard to their receptivity to the child's involvement with peers.

\section{8) Pride in the Child}

Healthy parents have pride in their children. This is one manifestation of their love. Such a parent's heart swells with pride when observing the child in school recitals, plays, and sporting events. Healthy parents may cry with joy on such occasions. They carry pictures in their wallets of their children and are quick to display these with pride to anyone who shows interest in looking at them. A reasonable degree of boasting about the child is also healthy and a manifestation of good parenting. Of course, when boasting is excessive or pride is being used in the service of denial of a child's obvious weaknesses, then this criterion is not satisfied.

\section{9) The Child's Own Stated Preference}

It is not by pure chance that this criterion is put near the bottom. Obviously, for younger children it is not a consideration. But even for older children it may be a risky indicator because the child's cognitive immaturity may result in his (her) stating preferences that are not really judicious, e.g., a six-year-old states, "I want to live with my daddy not my mommy. My daddy is a lot of fun. He buys me pizza and we play ball a lot. He lets me stay up and watch television. My mommy's mean. She makes me go to sleep early and makes me do homework." Furthermore, children who have been subjected to a program of alienation (Gardner, 1992, 1998) may profess preferences as a manifestation of their being programmed to denigrate a parent who might very well serve as a good and effective primary parent. 


\section{0) Commitment to the Care of the Handicapped Child}

Raising a handicapped child is one of the most sensitive tests of parenting capacity. A parent with compromised commitment is likely to exhibit such compromises when called upon to take on the extra tasks and burdens of rearing such a child. Obviously, then, preference should be given to the parent who exhibits greater commitment in such situations.

\section{CONCLUDING COMMENTS}

The examiner who focuses on these 20 areas, not superficially but in depth, is likely to be able to make reasonable parental preference recommendations in the vast majority of child-custody evaluations. In most cases the examiner will find that the parent for whom the evaluator recommends primary custody is likely to be superior in the majority of these indicators. It is important to note that I have studiously avoided giving any specific number of criteria that must be satisfied or any specific cutoff point. Rather, the examiner should operate on the principle that the greater the number of items on which a particular parent is superior the greater the justification for recommending that parent to be designated the primary custodial parent.

\section{REFERENCES}

Gardner, R.A. (1982), Family Evaluation in Child Custody Litigation. Cresskill, New Jersey: Creative Therapeutics, Inc.

(1986), Child Custody Litigation: A Guide for Parents and Mental Health Professionals. Cresskill, New Jersey: Creative Therapeutics, Inc.

(1989), Family Evaluation in Child Custody Mediation, Arbitration, and Litigation. Cresskill, New Jersey: Creative Therapeutics, Inc.

(1992), The Parental Alienation Syndrome. Cresskill, New Jersey: Creative Therapeutics, Inc.

(1998), The Parental Alienation Syndrome, Second Edition. Cresskill, New Jersey: Creative Therapeutics, Inc.

(1999), Guidelines for assessing parental preference in child-custody disputes. Journal of Divorce \& Remarriage, 30(1/2):1-9.

Michigan Guidelines for Assessing Parental Preference. MCL 722.23; MSA 25.312(3), as amended by PA 1993, No. 259, 1. effective November 1993. 